Brief paper

\title{
Loop detection of mobile robots using interval analysis
}

\author{
Clément Aubry a , Rozenn Desmare ${ }^{\mathrm{a}}$, Luc Jaulin ${ }^{\mathrm{b}, 1}$ \\ a IRENav, École navale, BP600, 29240 Brest Armées, France \\ b ENSTA Bretagne, LABSTICC, 2 rue François Verny, 29806 Brest, France
}

\section{A R T I C L E I N F O}

\section{Article history:}

Received 21 February 2012

Received in revised form

29 May 2012

Accepted 29 August 2012

Available online 8 December 2012

\section{Keywords:}

Loop closure

Interval analysis

Robotics

Tubes

SLAM

\begin{abstract}
A B S T R A C T
This paper proposes an original set-membership approach for loop detection of mobile robots in the situation where proprioceptive sensors only are available. To detect loops, the new concepts of the $t$-plane (which is a two dimensional space with time coordinates) are introduced. Intervals of functions (or tubes) are then used to represent uncertain trajectories and tests are provided in order to eliminate parts of the $t$-plane that do not correspond to any loop. An experiment with an actual underwater robot is proposed in order to illustrate the principle and the efficiency of the approach.
\end{abstract}

(C) 2012 Elsevier Ltd. All rights reserved.

\section{Introduction}

Creating loops and detecting them is an important topic for exploration (Lapierre, Zapata, \& Lépinay, 2007) and SLAM (Simultaneous Localization And Mapping) problems (Leonard \& Durrant-Whyte, 1992) where autonomous robots moving in an unknown environment have to build a map of this environment while simultaneously using this map to compute its location (Angel, Filliat, Doncieux, \& Meyer, 2008; Joly \& Rives, 2008). When the pose of the robot $\mathbf{p}(t)$ is perfectly known, loop detection amounts to finding all $t_{1}$ and $t_{2}$ such that $\mathbf{p}\left(t_{1}\right)=\mathbf{p}\left(t_{2}\right)$, with $t_{1} \neq$ $t_{2}$. Now, to prove the injectivity of a function, say $f(x)$, we generally search for the set of all pairs $\left(x_{1}, x_{2}\right)$ such that $f\left(x_{1}\right)=f\left(x_{2}\right)$ with $x_{1} \neq x_{2}$. If the resulting set is empty, then the function $f$ is proved to be injective. As a consequence, loop detection is close to the injectivity checking problem. In the control community, proving the injectivity has mainly been studied for identifiability analysis (see, e.g., Ben-Zvi (2010)) using computer algebra (Raksanyi, Lecourtier, Walter, \& Venot, 1985) or numerical methods (Braems, Jaulin, Kieffer, \& Walter, 2001; Lagrange, Delanoue, \& Jaulin, 2008) based on interval analysis. For our loop detection problem, proving

\footnotetext{
The material in this paper was not presented at any conference. This paper was recommended for publication in revised form by Associate Editor Michele Basseville under the direction of Editor Torsten Söderström.

E-mail addresses: clement.aubry@ecole-navale.fr (C. Aubry),

rozenn.desmare@ecole-navale.fr (R. Desmare), luc.jaulin@ensta-bretagne.fr (L. Jaulin).

${ }^{1}$ Tel.: +3302983489 10; fax: +330298348750.
}

the injectivity is even more difficult because the trajectory of the robot is badly known. The injectivity approach should then be adapted to take into account this uncertainty. This paper deals with loop detection in the case where the robot is only able to measure its velocity vector (which can be obtained from odometers, for instance) with a known bounded error (Meizel, Preciado-Ruiz, \& Halbwachs, 1996). This detection is made possible by introducing the new concept of the $t$-plane which contains pairs of times of the form $\left(t_{1}, t_{2}\right)$. A research on this $t$-plane with appropriate tests is proposed in order to characterize the set of all feasible loops.

Classically, SLAM techniques (Thrun, Bugard, \& Fox, 2005) use exteroceptive sensors only (such as lidars or cameras) to detect loops and then exploit the potential loop closures to get a better estimate of the trajectory. However, even if we are able to detect loops with proprioceptive sensors only, this detection cannot be used to refine the trajectory. Solving the problem of characterizing the set of all loops consistent with proprioceptive sensors, as treated in this paper, can help any conventional SLAM method by reducing the number of correspondences to be checked. Moreover, it can also be used to limit the number of false loop detections performed by classical SLAM approaches: a loop should now be consistent with both proprioceptive and exteroceptive observations.

The paper is organized as follows. Section 2 presents the loop detection problem and introduces the notion of $t$-space. Section 3 presents the basic notions on intervals and tubes. These notions will be used by Section 4 in order to build interval tests able to classify boxes covering the $t$-plane. Section 5 presents the algorithm for loop detections. A test-case related to an actual experiment is described in Sections 6 and 7 concludes the paper. 

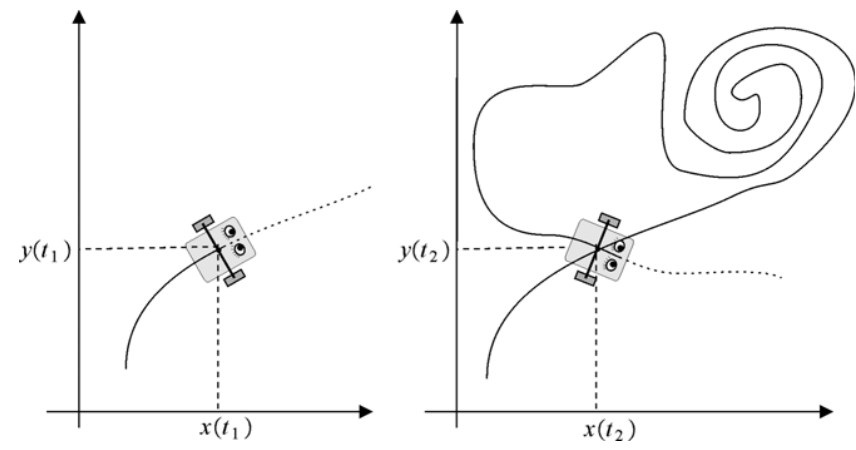

Fig. 1. A robot trajectory with one single loop.

\section{Proprioceptive loop detection}

Consider a robot (AUV, car, etc.) moving on a horizontal plane. Denote by $\mathbf{v}(t) \in \mathbb{R}^{2}$ its velocity vector which is assumed to be measured. The time $t$ belongs to the interval $\left[0, t_{\max }\right]$ associated to the duration of the mission. Define the function

$\mathbf{p}(t)=\int_{0}^{t} \mathbf{v}(\tau) d \tau$

which corresponds to the position of the robot's center on a frame, the origin of which corresponds to $\mathbf{p}(0)$. The two components of $\mathbf{p}$ are denoted by $(x, y)$. Define the loop set as

$\mathbb{T}^{*}=\left\{\left(t_{1}, t_{2}\right) \in\left[0, t_{\max }\right]^{2} \mid \mathbf{p}\left(t_{1}\right)=\mathbf{p}\left(t_{2}\right), t_{1}<t_{2}\right\}$,

or equivalently

$\mathbb{T}^{*}=\left\{\left(t_{1}, t_{2}\right) \in\left[0, t_{\mathrm{max}}\right]^{2} \mid \int_{t_{1}}^{t_{2}} \mathbf{v}(\tau) d \tau=\mathbf{0}, t_{1}<t_{2}\right\}$.

Fig. 1 presents a robot which performs a single loop. The set $\mathbb{T}^{*}$ has thus a single element which corresponds to $\mathbf{t}=\left(t_{1}, t_{2}\right)$. A vector $\mathbf{t}=\left(t_{1}, t_{2}\right)$ is called a $t$-pair. The set of all $t$-pairs is called the $t$-plane (see Fig. 2). For convenience, a $t$-pair in $\mathbb{T}^{*}$ will often be called a loop. Let us consider two loops $\mathbf{t}^{a}=\left(t_{1}^{a}, t_{2}^{a}\right)$ and $\mathbf{t}^{b}=$ $\left(t_{1}^{b}, t_{2}^{b}\right)$. If $\left[t_{1}^{a}, t_{2}^{a}\right] \subset\left[t_{1}^{b}, t_{2}^{b}\right]$, or equivalently, if $\mathbf{t}^{a}$ is inside the rightbottom quarter with vertex $\mathbf{t}^{b}$, then we say that the loop $\mathbf{t}^{b}$ encircles the loop $\mathbf{t}^{a}$. In the $t$-plane, $\mathbf{t}$ is a small loop if it encircles no other loop. Otherwise, it is a complex loop (i.e., a complex loop encircles some other loops). Assume that the robot is only able to measure the speed vector $\mathbf{v}$ with a known bounded error, i.e., a box $[\mathbf{v}](t)$ which contains $\mathbf{v}(t)$ is known for each $t \in\left[0, t_{\max }\right]$. The set of all feasible $\mathbf{t}=\left(t_{1}, t_{2}\right)$ is

$\mathbb{T}=\left\{\mathbf{t} \mid 0 \leq t_{1}<t_{2} \leq t_{\max }, \exists \mathbf{v} \in[\mathbf{v}], \int_{t_{1}}^{t_{2}} \mathbf{v}(\tau) d \tau=\mathbf{0}\right\}$

where $[\mathbf{v}]$ is an interval of functions (or tube) as defined in Section 3. The set $\mathbb{T}$ encloses $\mathbb{T}^{*}$. The main contribution of the paper is to provide an algorithm that is able to find an inner and an outer subpaving approximation of the set $\mathbb{T}$ in this machine interval experiment (Sainudiin, 2010) for loop detection. The algorithm will also be able to detect the number of loops by counting the number of elements of $\mathbb{T}^{*}$.

\section{Intervals and tubes}

Interval analysis (Chabert \& Jaulin, 2009; Moore, 1979) is composed of a set of numerical methods that make it possible to solve nonlinear problems such as localization (Drevelle \& Bonnifait, 2009), SLAM (Delahoche, Brassart, \& Clerentin, 2005), path planning (Delanoue, Jaulin, \& Cottenceau, 2006), control (Lydoire \& Poignet, 2003) or state estimation (Abdallah, Gning,
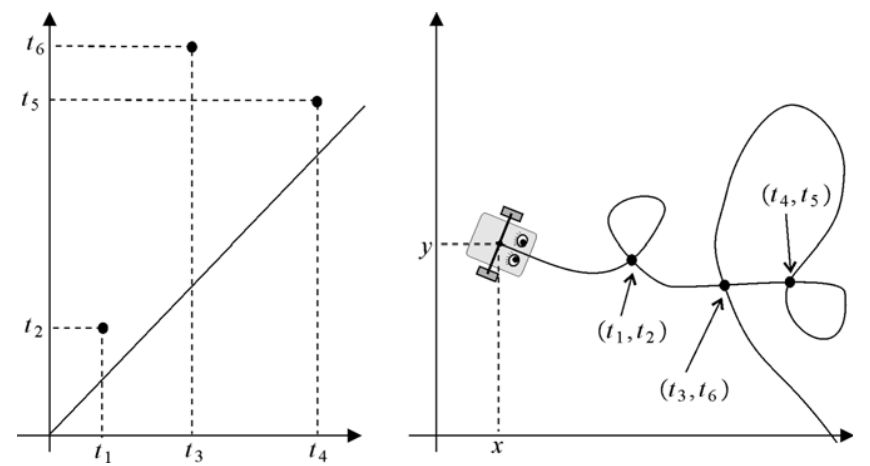

Fig. 2. Left: $t$-plane, with three loops composing $\mathbb{T}^{*}$; right: trajectory of the robot with two small loops $\left(t_{1}, t_{2}\right),\left(t_{4}, t_{5}\right)$ and one complex loop $\left(t_{3}, t_{6}\right)$. Note that $\left[t_{4}, t_{5}\right] \subset\left[t_{3}, t_{6}\right]$.

\& Bonnifait, 2008). The unknown variables could be Boolean numbers, integers, real numbers or functions. This section first introduces the notion of intervals in its more general form. Then, it presents the notion of tubes (or interval functions) and integrals of tubes that will be used later to solve our loop detection problem.

\subsection{Lattices}

A lattice $(\varepsilon, \leq)$ is a partially ordered set, closed under least upper and greatest lower bounds (see Davey and Priestley (2002), for more details). The least upper bound (or supremum) of $x$ and $y$ is called the join and is denoted by $x \vee y$. The greatest lower bound (or infimum) is called the meet and is written as $x \wedge y$.

Example. The set $\mathbb{R}^{n}$ is a lattice with respect to the partial order relation given by $\mathbf{x} \leq \mathbf{y} \Leftrightarrow \forall i \in\{1, \ldots, n\}, x_{i} \leq y_{i}$. We have $\mathbf{x} \wedge \mathbf{y}=\left(x_{1} \wedge y_{1}, \ldots, x_{n} \wedge y_{n}\right)$ and $\mathbf{x} \vee \mathbf{y}=\left(x_{1} \vee y_{1}, \ldots, x_{n} \vee y_{n}\right)$, where $x_{i} \wedge y_{i}=\min \left(x_{i}, y_{i}\right)$ and $x_{i} \vee y_{i}=\max \left(x_{i}, y_{i}\right)$.

A lattice $\mathcal{E}$ is complete if for all (finite of infinite) subsets $\mathcal{A}$ of $\mathscr{E}$, the least upper bound (denoted by $\wedge \mathcal{A}$ ) and the greatest lower bound (denoted $\vee \mathcal{A}$ ) belong to $\mathcal{E}$. When a lattice $\mathcal{E}$ is not complete, it is possible to add new elements (corresponding to the supremum or infimum of $\varepsilon$ ) to make it complete. For instance, the set $\mathbb{R}$ is not a complete lattice whereas $\overline{\mathbb{R}}=\mathbb{R} \cup\{-\infty, \infty\}$ is. By convention, for the empty set, we set $\wedge \emptyset=\vee \mathcal{E}$ and $\vee \emptyset=\wedge \mathscr{E}$. The product of two lattices $\left(\varepsilon_{1}, \leq_{1}\right)$ and $\left(\varepsilon_{2}, \leq_{2}\right)$ is the lattice $(\varepsilon, \leq)$ defined as the set of all $\left(a_{1}, a_{2}\right) \in \varepsilon_{1} \times \varepsilon_{2}$ with the order relation $\left(a_{1}, a_{2}\right) \leq\left(b_{1}, b_{2}\right) \Leftrightarrow\left(\left(a_{1} \leq_{1} b_{1}\right)\right.$ and $\left.\left(a_{2} \leq_{2} b_{2}\right)\right)$.

\subsection{Intervals}

A closed interval (or interval) $[x]$ of a complete lattice $\varepsilon$ is a subset of $\mathscr{E}$ which satisfies $[x]=\{x \in \mathcal{E} \mid \wedge[x] \leq x \leq \vee[x]\}$. Both $\emptyset$ and $\varepsilon$ are intervals of $\varepsilon$. An interval is a sub-lattice of $\varepsilon$. The set of all intervals of $\varepsilon$ is denoted by $\mathbb{I} \mathcal{E}$. An interval $[x]$ of $\varepsilon$ will also be denoted by $[x]=[\wedge[x], \vee[x]]_{\varepsilon}$. For example, the sets $\emptyset=$ $[\infty,-\infty]_{\mathbb{R}} ; \mathbb{R}=[-\infty, \infty]_{\mathbb{R}} ;[0,1]_{\mathbb{R}}$ and $[0, \infty]_{\mathbb{R}}$ are intervals of $\mathbb{R}$, the set $\{2,3,4,5\}=[2,5]_{\mathbb{N}}$ is an interval of the set of integers $\mathbb{N}$ and the set $\{4,6,8,10\}=[4,10]_{2 \mathbb{N}}$ is an interval of $2 \mathbb{N}$. The interval hull (or hull) [ $\mathcal{A}]$ of a subset $\mathcal{A}$ of $\mathcal{E}$ is the smallest interval of $\mathcal{E}$ which contains $\mathcal{A}$. If $[x]$ and $[y]$ are two intervals of $\mathcal{E}$, we denote by $[x] \sqcup[y]$ the interval hull of $[x] \cup[y]$. Given two complete lattices $\mathcal{D}, \mathcal{E}$ and a function $f: \mathcal{D} \rightarrow \mathcal{E}$. An inclusion function $[f]$ of $f$ is a function from $\mathbb{I} \mathscr{D}$ to $\mathbb{I} \mathscr{E}$ such that $\forall[x] \in \mathbb{I} \mathscr{D}, f([x]) \subset[f]([x])$. The inclusion function $[f]$ is thin, if $f([x])=[f]([x])$ when $[x]$ is a singleton (or a degenerated interval). Otherwise, $[f]$ is thick.

\subsection{Tubes}

We now introduce the notion of tubes that will be used for solving our loop closure problem. The set $\mathcal{F}^{n}$ of all functions from 


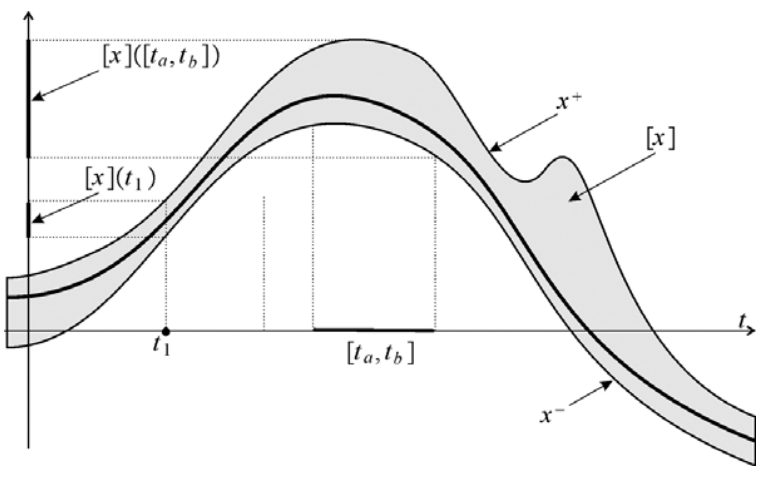

Fig. 3. A tube $[x]$ of $\mathbb{R}$ which encloses the function $x$.

$\mathbb{R}$ to $\overline{\mathbb{R}}^{n}$ is a complete lattice with the following partial order $\mathbf{x} \leq$ $\mathbf{y} \Leftrightarrow \forall t \in \mathbb{R}, \mathbf{x}(t) \leq \mathbf{y}(t)$. A tube [x] (see, e.g., Kurzhanski and Valyi (1997) and LeBars, Sliwka, Reynet, and Jaulin (2012)) is an interval $\left[\mathbf{x}^{-}, \mathbf{x}^{+}\right]$of $\mathcal{F}^{n}$, i.e., a pair of two functions $\mathbf{x}^{-}, \mathbf{x}^{+}$such that for all $t, \mathbf{x}^{-}(t) \leq \mathbf{x}^{+}(t)$. The set of all tubes of $\mathcal{F}^{n}$ is denoted by $\mathbb{I} \mathscr{F}^{n}$.

An element $\mathbf{x}$ of $\mathcal{F}^{n}$ belongs to the tube $[\mathbf{x}]$ if $\forall t, \mathbf{x}(t) \in[\mathbf{x}](t)$. Fig. 3 presents a function $x \in \mathcal{F}^{1}$ which is inside the tube $[x]$. This tube gives us information related to the unknown function $x$.

Interval evaluation of a tube. If $\mathbf{x}$ is a function from $\mathbb{R}$ to $\mathbb{R}^{n}$ (i.e., $\mathbf{x} \in \mathcal{F}^{n}$ ), we define $\mathbf{x}([t])=\{\mathbf{x}(t) \mid t \in[t]\}$. The interval evaluation of a tube $[\mathbf{x}]$ is defined by

$[\mathbf{x}]([t])=\bigsqcup_{t \in[t]}[\mathbf{x}](t)$,

i.e., $[\mathbf{x}]([t])$ is the smallest box which encloses all boxes $[\mathbf{x}](t), t \in$ $[t]$. It is easy to prove that $\mathbf{x} \in[\mathbf{x}], t \in[t] \Rightarrow \mathbf{x}(t) \in[\mathbf{x}]([t])$, and that no box smaller than $[\mathbf{x}]([t])$ satisfies this property.

Fast interval evaluation. As it will be the case in this paper, many interval evaluations of the tube $[\mathbf{x}]$ are required. In such a situation an efficient strategy is to compute for all $i \in\{1, \ldots, n\}$, the set $\mathcal{T}_{i}^{-}$ of all $t$ which correspond to local minimizers of the lower bound $x_{i}^{-}$ of $\left[x_{i}\right]$ and the set $\mathcal{T}_{i}^{+}$of all $t$ which correspond to local maximizers of the upper bound $x_{i}^{+}$of $\left[x_{i}\right]$.

Tube arithmetic. We can extend some classical operations we have on functions of $\mathcal{F}^{n}$, such as sums, multiplication, image by a function, ... to tubes (LeBars et al., 2012). It suffices to use the classical notion of interval arithmetic and inclusion functions (Moore, 1979) for all $t$. An arithmetic on tubes is thus a direct extension of interval arithmetic. As it is the case for interval computation, the result of an operation on tubes contains all results of the same operation performed on the enclosed elements of $\mathcal{F}^{n}$. Note that there also exist some techniques for the satisfaction of a state equation (Goldsztejn, Hayes, \& Collins, 2011; Raissi, Ramdani, \& Candau, 2004) that can be included in this arithmetic.

Integrals of tubes. Consider two numbers $t_{1}, t_{2}$ such that $t_{2} \geq$ $t_{1} \geq 0$. The integral of a tube $[\mathbf{x}]$ over an interval $\left[t_{1}, t_{2}\right]$ is defined by

$\int_{t_{1}}^{t_{2}}[\mathbf{x}](\tau) d \tau=\left\{\int_{t_{1}}^{t_{2}} \mathbf{x}(\tau) d \tau \mid \mathbf{x} \in[\mathbf{x}]\right\}$.

Since $t_{2} \geq t_{1}$, we deduce from the monotonicity of the integral operator that

$\int_{t_{1}}^{t_{2}}[\mathbf{x}](\tau) d \tau=\left[\int_{t_{1}}^{t_{2}} \mathbf{x}^{-}(\tau) d \tau, \int_{t_{1}}^{t_{2}} \mathbf{x}^{+}(\tau) d \tau\right]$.

From the definition of tube integrals, we have

$\mathbf{x} \in[\mathbf{x}] \Rightarrow \int_{t_{1}}^{t_{2}} \mathbf{x}(\tau) d \tau \in \int_{t_{1}}^{t_{2}}[\mathbf{x}](\tau) d \tau$.
Moreover, the interval primitive defined by $\int_{0}^{t}[\mathbf{x}](\tau) d \tau$ defines a tube that vanishes for $t=0$.

Integrals with interval bounds. Assume now that the positive bounds $t_{1}, t_{2}$ of the interval $\left[t_{1}, t_{2}\right]$ are uncertain, or more precisely, we only know that $t_{1}, t_{2}$ belong to the intervals $\left[t_{1}\right],\left[t_{2}\right]$. The following theorem makes it possible to compute the smallest interval which encloses all feasible values for the integral $\int_{t_{1}}^{t_{2}} \mathbf{x}(\tau) d \tau$. To the best of our knowledge, this theorem is proposed here for the first time.

Theorem 1. Consider a function $\mathbf{x}$ from $\mathbb{R} \rightarrow \mathbb{R}^{n}$ and $t_{1}, t_{2} \in \mathbb{R}$. Assume that $t_{2} \geq t_{1} \geq 0, t_{1} \in\left[t_{1}\right], t_{2} \in\left[t_{2}\right], \mathbf{x} \in[\mathbf{x}]$. Then the smallest box which encloses all feasible values for $\int_{t_{1}}^{t_{2}} \mathbf{x}(\tau) d \tau$ is given by

$$
\begin{aligned}
\int_{\left[t_{1}\right]}^{\left[t_{2}\right]}[\mathbf{x}](\tau) d \tau= & {\left[\mathrm{lb}\left(\mathbf{y}^{-}\left(\left[t_{2}\right]\right)-\mathbf{y}^{-}\left(\left[t_{1}\right]\right)\right),\right.} \\
& \left.\times \operatorname{ub}\left(\mathbf{y}^{+}\left(\left[t_{2}\right]\right)-\mathbf{y}^{+}\left(\left[t_{1}\right]\right)\right)\right],
\end{aligned}
$$

where $[\mathbf{y}](t)=\int_{0}^{t}[\mathbf{x}](\tau) d \tau$ is the interval primitive of $[\mathbf{x}]$.

Proof. Consider the $i$ th component of the integral and let us try to find its lower bound $z_{i}^{-}$. It is given by

$z_{i}^{-}=\min \left\{\int_{t_{1}}^{t_{2}} x_{i}(\tau) d \tau \mid t_{1} \in\left[t_{1}\right], t_{2} \in\left[t_{2}\right], x_{i} \in\left[x_{i}\right]\right\}$.

Since the integral operator is monotonic, the optimal contribution of function $x_{i}$ is obtained for $x_{i}=\mathrm{lb}\left(\left[x_{i}\right]\right)=x_{i}^{-}$, we get

$z_{i}^{-}=\min \left\{\int_{t_{1}}^{t_{2}} x_{i}^{-}(\tau) d \tau \mid t_{1} \in\left[t_{1}\right], t_{2} \in\left[t_{2}\right]\right\}$.

Now, the $i$ th lower bound $y_{i}^{-}(t)$ of $[\mathbf{y}](t)$ corresponds to $\int_{0}^{t}$ $x_{i}^{-}(\tau) d \tau$, the primitive of $x_{i}^{-}$that vanishes for $t=0$. Thus, we get

$z_{i}^{-}=\min \left\{y_{i}^{-}\left(t_{2}\right)-y_{i}^{-}\left(t_{1}\right) \mid t_{1} \in\left[t_{1}\right], t_{2} \in\left[t_{2}\right]\right\}$.

Since $t_{1}$ and $t_{2}$ occur only once in the expression $y_{i}^{-}\left(t_{2}\right)-y_{i}^{-}\left(t_{1}\right)$, we can write

$z_{i}^{-}=\min \left\{y_{i}^{-}\left(\left[t_{2}\right]\right)-y_{i}^{-}\left(\left[t_{1}\right]\right)\right\}=\operatorname{lb}\left(y_{i}^{-}\left(\left[t_{2}\right]\right)-y_{i}^{-}\left(\left[t_{1}\right]\right)\right)$.

Using a similar reasoning for the upper bound $z_{i}^{+}$of the $i$ th component of the integral, we get $z_{i}^{+}=\mathrm{ub}\left(y_{i}^{+}\left(\left[t_{2}\right]\right)-y_{i}^{+}\left(\left[t_{1}\right]\right)\right)$, which terminates the proof.

Example. Consider the tube defined by $[\mathbf{x}](t)=[t-2,2 t+3]$, with $t \geq 0$. Take $\left[t_{1}\right]=[1,4],\left[t_{2}\right]=[5,6]$ and let us compute

$I=\int_{\left[t_{1}\right]}^{\left[t_{2}\right]}[\mathbf{x}](\tau) d \tau=\int_{[1,4]}^{[5,6]}[\tau-2,2 \tau+3] d \tau$.

From Theorem 1, we have

$I=\left[\mathrm{lb}\left(y^{-}\left(\left[t_{2}\right]\right)-y^{-}\left(\left[t_{1}\right]\right)\right), \mathrm{ub}\left(y^{+}\left(\left[t_{2}\right]\right)-y^{+}\left(\left[t_{1}\right]\right)\right)\right]$.

The primitive tube $[\mathbf{y}]$ is

$$
\begin{aligned}
{[\mathbf{y}](t) } & =\int_{0}^{t}[\mathbf{x}](\tau) d \tau=\int_{0}^{t}[\tau-2,2 \tau+3] d \tau \\
& =\left[\int_{0}^{t}(\tau-2) d \tau, \int_{0}^{t}(2 \tau+3) d \tau\right] \\
& =\left[\frac{1}{2} t^{2}-2 t, t^{2}+3 t\right]=\left[y^{-}(t), y^{+}(t)\right] .
\end{aligned}
$$




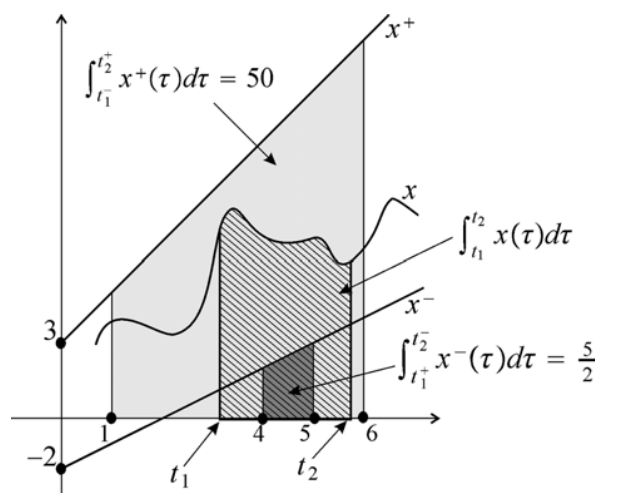

Fig. 4. Illustration of the integral of a tube over interval bounds.

Since for our example both bounds $y^{-}(t), y^{+}(t)$ are increasing, we have

$y^{-}\left(\left[t_{1}\right]\right)=\left[\frac{1}{2}\left(t_{1}^{-}\right)^{2}-2 t_{1}^{-}, \frac{1}{2}\left(t_{1}^{+}\right)^{2}-2 t_{1}^{+}\right]=\left[-\frac{3}{2}, 0\right]$

$y^{+}\left(\left[t_{1}\right]\right)=\left[\left(t_{1}^{-}\right)^{2}+3 t_{1}^{-},\left(t_{1}^{+}\right)^{2}+3 t_{1}^{+}\right]=[4,28]$

$y^{-}\left(\left[t_{2}\right]\right)=\left[\frac{1}{2}\left(t_{2}^{-}\right)^{2}-2 t_{2}^{-}, \frac{1}{2}\left(t_{2}^{+}\right)^{2}-2 t_{2}^{+}\right]=\left[\frac{5}{2}, 6\right]$

$y^{+}\left(\left[t_{2}\right]\right)=\left[\left(t_{2}^{-}\right)^{2}+3 t_{2}^{-},\left(t_{2}^{+}\right)^{2}+3 t_{2}^{+}\right]=[40,54]$.

Thus

$y^{-}\left(\left[t_{2}\right]\right)-y^{-}\left(\left[t_{1}\right]\right)=\left[\frac{5}{2}, 6\right]-\left[-\frac{3}{2}, 0\right]=\left[\frac{5}{2}, \frac{15}{2}\right]$

$y^{+}\left(\left[t_{2}\right]\right)-y^{+}\left(\left[t_{1}\right]\right)=[40,54]-[4,28]=[12,50]$.

Finally

$$
\begin{aligned}
I & =\left[\mathrm{lb}\left(y^{-}\left(\left[t_{2}\right]\right)-y^{-}\left(\left[t_{1}\right]\right)\right), \mathrm{ub}\left(y^{+}\left(\left[t_{2}\right]\right)-y^{+}\left(\left[t_{1}\right]\right)\right)\right] \\
& =\left[\mathrm{lb}\left(\left[\frac{5}{2}, \frac{15}{2}\right]\right), \mathrm{ub}([12,50])\right]=\left[\frac{5}{2}, 50\right] .
\end{aligned}
$$

Fig. 4 illustrates that for unknown $t_{1}, t_{2}, x$, we have

$\left\{\begin{array}{l}t_{1} \in\left[t_{1}\right] \\ t_{2} \in\left[t_{2}\right] \\ x \in[x]\end{array} \Rightarrow \int_{t_{1}}^{t_{2}} x(\tau) d \tau \in \int_{\left[t_{1}\right]}^{\left[t_{2}\right]}[x](\tau) d \tau\right.$.

Note also that for this particular case,

$\int_{\left[t_{1}\right]}^{\left[t_{2}\right]}[x](\tau) d \tau=\left[\int_{4}^{5}(\tau-2) d \tau, \int_{1}^{6}(2 t+3) d t\right]$.

Fast evaluation of interval integrals. Assume that we have to compute the interval integral $\int_{\left[t_{1}\right]}^{\left[t_{2}\right]}[\mathbf{x}](\tau) d \tau$ for many different intervals $\left[t_{1}\right]$ and $\left[t_{2}\right]$ (as it will be the case latter in this paper to solve the loop detection problem). We first compute the interval primitive

$[\mathbf{y}](t)=\int_{0}^{t}[\mathbf{x}](\tau) d \tau=\left[\int_{0}^{t} \mathbf{x}^{-}(\tau) d \tau, \int_{0}^{t} \mathbf{x}^{+}(\tau) d \tau\right]$.

Since $[\mathbf{y}](t)$ does not depend on $\left[t_{1}\right]$ and $\left[t_{2}\right]$, the calculus has to be performed only once. In a second step we have to compute fast evaluations of $\mathbf{y}^{-}\left(\left[t_{1}\right]\right), \mathbf{y}^{+}\left(\left[t_{1}\right]\right), \mathbf{y}^{-}\left(\left[t_{2}\right]\right), \mathbf{y}^{+}\left(\left[t_{2}\right]\right)$. Finally, the optimal interval integral is obtained from the formula (6).

\subsection{Newton operator}

Consider a smooth function $\mathbf{f}: \mathbb{R}^{n} \rightarrow \mathbb{R}^{n}$ and denote by $\mathbf{J f}_{\mathbf{f}}$ its Jacobian matrix. The Newton operator (Moore, 1979) is the operator from $\mathbb{\mathbb { R } ^ { n }}$ to $\mathbb{R}^{n}$ defined by

$\mathcal{N}\left(\mathbf{f},\left[\mathbf{J}_{\mathbf{f}}\right],[\mathbf{x}]\right)=\widehat{\mathbf{x}}-\left[\mathbf{J}_{\mathbf{f}}\right]^{-1}([\mathbf{x}]) \cdot \mathbf{f}(\widehat{\mathbf{x}})$,

where $\left[\mathbf{J}_{\mathbf{f}}\right]$ is an inclusion function of $\mathbf{J}_{\mathbf{f}}$ and where $\widehat{\mathbf{x}}$ is the center of $[\mathbf{x}]$. We know from Moore (Moore, 1979) that

$\mathcal{N}\left(\mathbf{f},\left[\mathbf{J}_{\mathbf{f}}\right],[\mathbf{x}]\right) \subset[\mathbf{x}] \Rightarrow \exists ! \mathbf{x} \in[\mathbf{x}], \quad \mathbf{f}(\mathbf{x})=\mathbf{0}$,

where $\exists$ ! means 'there exists a unique'. For some applications (as it will be the case for our loop detection problem), $\mathbf{f}$ is not exactly known, but only a thick inclusion function [f] of $\mathbf{f}$ is available. In such a case, since $\mathbf{f} \in[\mathbf{f}]$, we obviously have

$\mathcal{N}\left([\mathbf{f}],\left[\mathbf{J}_{\mathbf{f}}\right],[\mathbf{x}]\right) \subset[\mathbf{x}] \Rightarrow \exists ! \mathbf{x} \in[\mathbf{x}], \quad \mathbf{f}(\mathbf{x})=\mathbf{0}$

where $\left.\mathcal{N}\left([\mathbf{f}],\left[\mathbf{J}_{\mathbf{f}}\right],[\mathbf{x}]\right)=\widehat{\mathbf{x}}-\left[\mathbf{J}_{\mathbf{f}}\right]^{-1}([\mathbf{x}]) \cdot[\mathbf{f}] \widehat{\mathbf{x}}\right)$. Equivalently, we could write

$\mathcal{N}\left([\mathbf{f}],\left[\mathbf{J}_{\mathbf{f}}\right],[\mathbf{x}]\right) \subset[\mathbf{x}] \Rightarrow \forall \mathbf{f} \in[\mathbf{f}], \quad \exists ! \mathbf{x} \in[\mathbf{x}], \quad \mathbf{f}(\mathbf{x})=\mathbf{0}$.

\section{Tests}

The loop set $\mathbb{T}^{*}$ is composed of a finite set of points of the $t$-plane. The set $\mathbb{T}$ of all feasible $\mathbf{t}$, defined by (4), encloses $\mathbb{T}^{*}$. Consider a $t$-box $[\mathbf{t}]$ of the $t$-plane. If $[\mathbf{t}] \subset \mathbb{T}$, it is said to be feasible. If $[\mathbf{t}] \cap \mathbb{T}=\emptyset$, it is unfeasible; otherwise, it is undetermined. In some situations, we can prove that [t $\mathbf{t}$ contains a single point of $\mathbb{T}^{*}$. This section proposes different tests that will be used by the main algorithm in order to classify boxes $[\mathbf{t}]$ of the $t$-plane.

\subsection{Integral test}

Proposition 1. Given a $t$-box $[\mathbf{t}]=\left[t_{1}\right] \times\left[t_{2}\right]$, we have

$\left.\begin{array}{c}{\left[t_{1}\right]-\left[t_{2}\right] \subset \mathbb{R}^{-} \text {and }} \\ \int_{\left[t_{1}\right]}^{\left[t_{2}\right]} \mathbf{v}^{-}(\tau) d \tau \leq \mathbf{0} \leq \int_{\left[t_{1}\right]}^{\left[t_{2}\right]} \mathbf{v}^{+}(\tau) d \tau\end{array}\right\} \Rightarrow[\mathbf{t}] \subset \mathbb{T}$,

and

$\left.\begin{array}{l}{\left[t_{1}\right]-\left[t_{2}\right] \subset \mathbb{R}^{+} \text {or }} \\ \mathbf{0} \notin \int_{\left[t_{1}\right]}^{\left[t_{2}\right]}[\mathbf{v}](\tau) d \tau\end{array}\right\} \Rightarrow[\mathbf{t}] \cap \mathbb{T}=\emptyset$.

Proof. From (4), $\mathbf{t} \in \mathbb{T}$ if and only if (i) $t_{1}-t_{2}<0$ and (ii) $\exists \mathbf{v} \in[\mathbf{v}], \int_{t_{1}}^{t_{2}} \mathbf{v}(\tau) d \tau=\mathbf{0}$. Now, condition (ii) is equivalent to

$$
\begin{aligned}
& \left\{\begin{array}{l}
\exists v_{1} \in\left[v_{1}\right], \quad \int_{t_{1}}^{t_{2}} v_{1}(\tau) d \tau=0 \\
\exists v_{2} \in\left[v_{2}\right], \quad \int_{t_{1}}^{t_{2}} v_{2}(\tau) d \tau=0 \\
\Leftrightarrow\left\{\begin{array}{l}
\int_{t_{1}}^{t_{2}} v_{1}^{-}(\tau) d \tau \leq 0 \quad \text { and } \int_{t_{1}}^{t_{2}} v_{1}^{+}(\tau) d \tau \geq 0 \\
\int_{t_{1}}^{t_{2}} v_{2}^{-}(\tau) d \tau \leq 0 \quad \text { and } \int_{t_{1}}^{t_{2}} v_{2}^{+}(\tau) d \tau \geq 0
\end{array}\right. \\
\Leftrightarrow \int_{t_{1}}^{t_{2}} \mathbf{v}^{-}(\tau) d \tau<\mathbf{0}, \int_{t_{1}}^{t_{2}} \mathbf{v}^{+}(\tau) d \tau>\mathbf{0} .
\end{array}\right.
\end{aligned}
$$

\subsection{Injectivity test}

When $\left[t_{1}\right] \cap\left[t_{2}\right] \neq \emptyset$, the integral test will not be able to reject a $t$-box, even if it has a small width. Now, if inside the interval 
$\left[t_{1}^{-}, t_{2}^{+}\right]$, the function $\mathbf{p}(t)$ as defined by (1) is injective, we will not be able to find $t_{1} \in\left[t_{1}\right]$ and $t_{2} \in\left[t_{2}\right]$ such that $\mathbf{p}\left(t_{1}\right)=\mathbf{p}\left(t_{2}\right)$. And thus the box $\left[t_{1}\right] \times\left[t_{2}\right]$ will be outside $\mathbb{T}$. The following proposition, based on this local injectivity condition, will be used to possibly reject such $t$-boxes.

Proposition 2. Given a $t$-box $[\mathbf{t}]=\left[t_{1}\right] \times\left[t_{2}\right]$, we have

$\left(\mathbf{0} \notin[\mathbf{v}]\left(\left[t_{1}^{-}, t_{2}^{+}\right]\right)\right) \Rightarrow[\mathbf{t}] \cap \mathbb{T}=\emptyset$.

Proof. In order to show the theorem by contradiction, let us suppose that $\mathbf{0} \notin[\mathbf{v}]\left(\left[t_{1}^{-}, t_{2}^{+}\right]\right)$and $[\mathbf{t}] \cap \mathbb{T} \neq \emptyset$. Take $\left(t_{1}, t_{2}\right) \in$ $[\mathbf{t}] \cap \mathbb{T}$, from (4), we have

$\exists \mathbf{v} \in[\mathbf{v}], \quad \int_{t_{1}}^{t_{2}} \mathbf{v}(\tau) d \tau=\mathbf{0}$.

The function $\mathbf{q}(t)=\int_{0}^{t} \mathbf{v}(\tau) d \tau$ (see (1)) is differentiable with respect to $t$. We can thus apply the generalized mean value theorem (Lagrange, Delanoue, \& Jaulin, 2007) on the interval $\left[t_{1}^{-}, t_{2}^{+}\right]$. We get that if $t_{1}, t_{2} \in\left[t_{1}^{-}, t_{2}^{+}\right]$, then

$\exists \overline{\mathbf{v}} \in\left[\frac{d \mathbf{q}}{d t}\left(\left[t_{1}^{-}, t_{2}^{+}\right]\right)\right], \quad \mathbf{q}\left(t_{2}\right)-\mathbf{q}\left(t_{1}\right)=\overline{\mathbf{v}} \cdot\left(t_{2}-t_{1}\right)$,

where $\left[\frac{d \mathbf{q}}{d t}\left(\left[t_{1}^{-}, t_{2}^{+}\right]\right)\right]$denotes any box enclosing the set $\left\{\frac{d \mathbf{q}}{d t}(t) \mid t\right.$ $\left.\in\left[t_{1}^{-}, t_{2}^{+}\right]\right\}$. Now, from (7), we have $\mathbf{q}\left(t_{2}\right)-\mathbf{q}\left(t_{1}\right)=\mathbf{0}$ and $\frac{d \mathbf{q}}{d t}(t)=$ $\mathbf{v}(t)$. Thus (8) implies that $\exists \overline{\mathbf{v}} \in[\mathbf{v}]\left(\left[t_{1}^{-}, t_{2}^{+}\right]\right), \mathbf{0}=\overline{\mathbf{v}} \cdot\left(t_{2}-t_{1}\right)$, i.e., $\exists \overline{\mathbf{v}} \in[\mathbf{v}]\left(\left[t_{1}^{-}, t_{2}^{+}\right]\right), \overline{\mathbf{v}}=\mathbf{0}$ which is in contradiction with the supposition that $\mathbf{0} \notin[\mathbf{v}]\left(\left[t_{1}^{-}, t_{2}^{+}\right]\right)$.

\subsection{Newton test}

Using the Newton test for proving unicity properties in the context of dynamical systems has been first proposed by Tucker (1999) to prove the existence of the Lorenz attractor.

Proposition 3. Given a $t$-box $[\mathbf{t}]=\left[t_{1}\right] \times\left[t_{2}\right]$, such that $\left[t_{1}\right]-\left[t_{2}\right] \subset$ $\mathbb{R}^{-}$, if

$\hat{\mathbf{t}}-\left(-[\mathbf{v}]\left(\left[t_{1}\right]\right) \quad[\mathbf{v}]\left(\left[t_{2}\right]\right)\right)^{-1} \cdot \int_{\hat{t}_{1}}^{\hat{t}_{2}}[\mathbf{v}](\tau) d \tau \subset[\mathbf{t}]$,

then, there exists a unique $\mathbf{t} \in[\mathbf{t}]$ such that $\mathbf{t} \in \mathbb{T}^{*}$.

Proof. Take a box $[\mathbf{t}]=\left[t_{1}\right] \times\left[t_{2}\right]$ such that $\left[t_{2}\right]-\left[t_{1}\right] \subset \mathbb{R}^{+}$. From (3), we have $\left(t_{1}, t_{2}\right) \in \mathbb{T}^{*} \cap[\mathbf{t}] \Leftrightarrow \exists \mathbf{t} \in[\mathbf{t}], \mathbf{f}(\mathbf{t})=\mathbf{0}$, where $\mathbf{f}(\mathbf{t})=\int_{t_{1}}^{t_{2}} \mathbf{v}(\tau) d \tau, \mathbf{t}=\left(t_{1}, t_{2}\right)$. The Newton operator can prove that there exists a unique $\mathbf{t} \in[\mathbf{t}]$ which belongs to $\mathbb{T}^{*}$. More precisely, from Section 3.4, we have

$\mathcal{N}\left([\mathbf{f}],\left[\mathbf{J}_{\mathbf{f}}\right],[\mathbf{t}]\right) \subset[\mathbf{t}] \Rightarrow \exists ! \mathbf{t} \in[\mathbf{t}], \quad \mathbf{f}(\mathbf{t})=\mathbf{0}$,

where $\mathcal{N}\left([\mathbf{f}],\left[\mathbf{J}_{\mathbf{f}}\right],[\mathbf{t}]\right)=\widehat{\mathbf{t}}-\left[\mathbf{J}_{\mathbf{f}}\right]^{-1}([\mathbf{t}]) \cdot[\mathbf{f}](\widehat{\mathbf{t}})$. In our context, since

$\mathbf{J}_{\mathbf{f}}(\mathbf{t})=\left(\frac{\partial \mathbf{f}}{\partial t_{1}}(\mathbf{t}) \quad \frac{\partial \mathbf{f}}{\partial t_{2}}(\mathbf{t})\right)=\left(\begin{array}{ll}-\mathbf{v}\left(t_{1}\right) & \mathbf{v}\left(t_{2}\right)\end{array}\right)$,

the Newton operator to be used is

$\mathcal{N}([\mathbf{v}],[\mathbf{t}])=\hat{\mathbf{t}}-\left(-[\mathbf{v}]\left(\left[t_{1}\right]\right) \quad[\mathbf{v}]\left(\left[t_{2}\right]\right)\right)^{-1} \cdot \int_{\hat{t}_{1}}^{\hat{t}_{2}}[\mathbf{v}](\tau) d \tau$.

If $\mathcal{N}([\mathbf{v}],[\mathbf{t}]) \subset$ [t], then there exists a unique $t$-pair $\left(t_{1}, t_{2}\right) \in[\mathbf{t}]$ that belongs to $\mathbb{T}^{*}$.

Remark. Even if the Newton test $\mathcal{N}([\mathbf{v}],[\mathbf{t}]) \subset[\mathbf{t}]$ is true, the set of feasible $\mathbf{t}=\left(t_{1}, t_{2}\right)$ in [t] defined by
Table 1

Algorithm for detecting loops made by a mobile robot.

\begin{tabular}{ll}
\hline Algorithm Loop $\left(\right.$ in: $\varepsilon, t_{\max },[\mathbf{v}] ;$ out: $\left.\mathbb{T}^{\text {in }}, \mathbb{T}^{?}, \mathbb{T}^{\text {out }}\right)$ \\
\hline 1 & $\begin{array}{l}\mathcal{Q}:=\left\{\left[0, t_{\max }\right] \times\left[0, t_{\max }\right]\right\} ; \mathbb{T}^{\text {in }}=\emptyset ; \mathbb{T}^{?}=\emptyset ; \mathbb{T}^{\text {out }}=\emptyset ; \\
\text { If } \mathcal{Q} \neq \emptyset \text {, take an element }[\mathbf{t}] \text { in } \mathcal{Q} ; \text { else return. }\end{array}$ \\
3 & If $\left\{\begin{array}{l}{\left[t_{1}\right]-\left[t_{2}\right] \subset \mathbb{R}^{+} \text {or }} \\
\mathbf{0} \notin \int_{\left[t_{1}\right]}^{\left[t_{2}\right]}[\mathbf{v}](\tau) d \tau \text { or, then } \mathbb{T}^{\text {out }}:=\mathbb{T}^{\text {out }} \cup[\mathbf{t}] ; \text { go to } 2 ; \\
\mathbf{0} \notin[\mathbf{v}]\left(\left[t_{1}^{-}, t_{2}^{+}\right]\right)\end{array}\right.$ \\
4 & If $\left\{\begin{array}{l}{\left[t_{1}\right]-\left[t_{2}\right] \subset \mathbb{R}^{-} \text {and }} \\
\int_{\left[t_{1}\right]}^{\left[t_{2}\right]} \mathbf{v}^{-}(\tau) d \tau \leq \mathbf{0} \leq \int_{\left[t_{1}\right]}^{\left[t_{2}\right]} \mathbf{v}^{+}(\tau) d \tau, \\
5\end{array}\right.$ \\
then $\mathbb{T}^{\text {in }}:=\mathbb{T}^{\text {in }} \cup[\mathbf{t}] ;$ go to $2 ;$ \\
If width $([\mathbf{t}])<\varepsilon$, then $\mathbb{T}^{?}:=\mathbb{T}^{?} \cup[\mathbf{t}] ;$ go to $2 ;$ \\
Bisect $[\mathbf{t}]$ and store the resulting boxes in $\mathcal{Q} ;$ go to 2.
\end{tabular}

$\hat{\mathbb{T}}([\mathbf{v}],[\mathbf{t}])=\bigcup_{\mathbf{v} \in[\mathbf{v}]}\left\{\left(t_{1}, t_{2}\right) \in[\mathbf{t}] \mid \int_{t_{1}}^{t_{2}} \mathbf{v}(\tau) d \tau=\mathbf{0}\right\}$

encloses an infinite number of elements. Now, for all feasible velocity functions $\mathbf{v} \in[\mathbf{v}]$, the set

$\hat{\mathbb{T}}(\mathbf{v},[\mathbf{t}])=\left\{\left(t_{1}, t_{2}\right) \in[\mathbf{t}] \mid \int_{t_{1}}^{t_{2}} \mathbf{v}(\tau) d \tau=\mathbf{0}\right\}$

has a unique element. This is also the case for the true $\mathbf{v}$, since it belongs to $[\mathbf{v}]$. As a consequence, the Newton test proves that there exists a single loop corresponding to [t] , even if there exist an infinite number of feasible loops.

\subsection{Summary}

Given a $t$-box [t $\mathbf{t}]$, a $t$-pair $\hat{t} \in[\mathbf{t}]$, we have the following tests

$$
\begin{aligned}
& {\left[t_{1}\right]-\left[t_{2}\right] \subset \mathbb{R}^{+} \text {or }} \\
& \left.\begin{array}{l}
\mathbf{0} \notin \int_{\left[t_{1}\right]}^{\left[t_{2}\right]}[\mathbf{v}](\tau) d \tau \text { or } \\
\mathbf{0} \notin[\mathbf{v}]\left(\left[t_{1}^{-}, t_{2}^{+}\right]\right)
\end{array}\right\} \Rightarrow[\mathbf{t}] \cap \mathbb{T}=\emptyset, \\
& {\left[t_{1}\right]-\left[t_{2}\right] \subset \mathbb{R}^{-} \text {and }} \\
& \left.\int_{\left[t_{1}\right]}^{\left[t_{2}\right]} \mathbf{v}^{-}(\tau) d \tau \leq \mathbf{0} \leq \int_{\left[t_{1}\right]}^{\left[t_{2}\right]} \mathbf{v}^{+}(\tau) d \tau\right\} \Rightarrow[\mathbf{t}] \subset \mathbb{T}, \\
& {\left[t_{1}\right]-\left[t_{2}\right] \subset \mathbb{R}^{-} \text {and }} \\
& \left.\begin{array}{c}
\hat{\mathbf{t}}-\left(-[\mathbf{v}]\left(\left[t_{1}\right]\right) \quad[\mathbf{v}]\left(\left[t_{2}\right]\right)\right)^{-1} \\
\cdot \int_{\hat{t}_{1}}^{\hat{t}_{2}}[\mathbf{v}](\tau) d \tau \subset[\mathbf{t}]
\end{array}\right\} \Rightarrow \exists ! \mathbf{t} \in[\mathbf{t}] \cap \mathbb{T}^{*} .
\end{aligned}
$$

\section{Algorithm}

The algorithm to be proposed to characterize the set $\mathbb{T}^{*}$ is a branch and bound algorithm similar to SIVIA (Set Inverter Via Interval Analysis) (Jaulin, Kieffer, Didrit, \& Walter, 2001). The main idea of the algorithm is to partition the $t$-plane with non overlapping boxes and to classify all boxes using the tests developed in Section 4. The input of the algorithm is the tube [v], the accuracy $\varepsilon$ and the duration of the mission $t_{\max }$. The algorithm returns three subpavings (i.e., union of boxes): $\mathbb{T}^{\text {in }}$ which encloses boxes [t] which have been proved to be inside $\mathbb{T} ; \mathbb{T}^{\text {out }}$ which encloses $[\mathbf{t}]$ which are outside $\mathbb{T}$ and $\mathbb{T}^{\text {? }}$ which contains small boxes [t] for which nothing is known. The algorithm is given by Table 1 . 


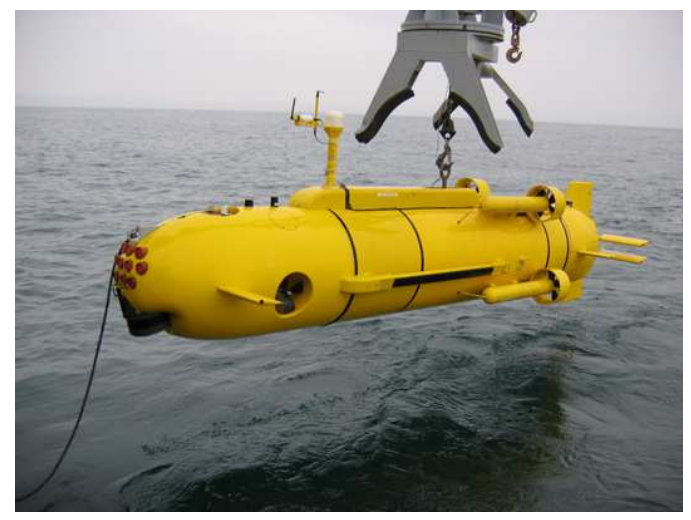

Fig. 5. The autonomous underwater vehicle, Redermor, built by the GESMA (Groupe d'Etude Sous-Marine de l'Atlantique).

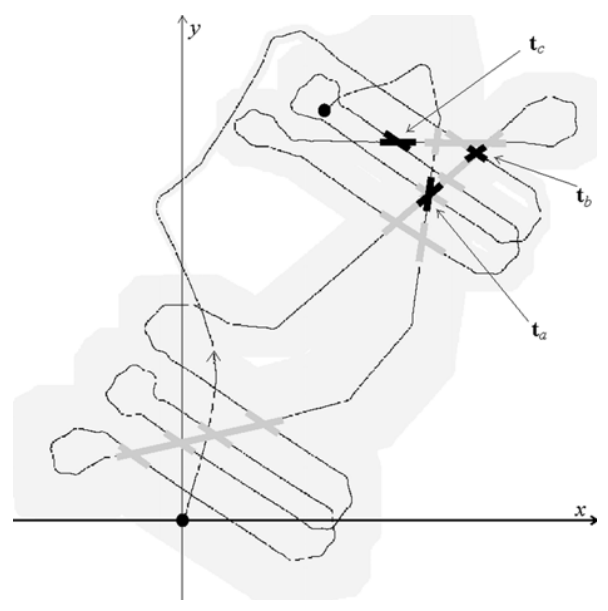

Fig. 6. Tube (painted gray) enclosing the trajectory $\mathbf{p}$ of the robot. The 14 black/gray crosses correspond to detected loops.

At Step 1, the list $\mathcal{Q}$ is initialized with a single box which corresponds to the search box in the $t$-plane. At Step 2, a $t$-box is taken from $\mathcal{Q}$, if $\mathcal{Q}$ is non-empty. If the list is empty, the algorithm terminates. Steps 3 and 4 try to show that the current $t$-box [t] is outside or inside $\mathbb{T}$ using the tests developed in Section 4 . If one of the tests succeeds, another box is taken in the list $\mathcal{Q}$ at Step 2. If the current $t$-box [t] is too small, the algorithm stores it into $\mathbb{T}^{\text {? }}$ at Step 5 and $[\mathbf{t}]$ will not be bisected anymore. If all tests failed and if [t] is still large enough, it is bisected at Step 6 into two boxes that are stored in the current list $\mathcal{Q}$. When the algorithm terminates, we have (Jaulin \& Walter, 1993)

$\mathbb{T}^{\text {in }} \subset \mathbb{T} \subset\left(\mathbb{T}^{\text {in }} \cup \mathbb{T}^{?}\right)$.

To detect loops, we take all boxes $[\mathbf{t}]$ stored inside $\mathbb{T}^{\text {in }}$. Then, we apply an epsilon-inflation (Moore, 1979), and we apply the Newton test presented in Section 4.3. All boxes [t] for which the Newton test succeeds are stored inside a list $\mathbb{T}^{N}$. Note that without the epsilon inflation the Newton test would always fail. Now, this inflation generates overlapping boxes corresponding to identical loops. These redundant detections could be deleted by replacing all pairs $\left(\left[\mathbf{t}^{a}\right],\left[\mathbf{t}^{b}\right]\right)$ of overlapping boxes in $\mathbb{T}^{N}$ by their intersection $\left[\mathbf{t}^{a}\right] \cap\left[\mathbf{t}^{b}\right]$, until no more overlapping boxes exists in $\mathbb{T}^{N}$.

\section{Test case}

Consider an underwater robot moving on a horizontal plane described by the following state equations

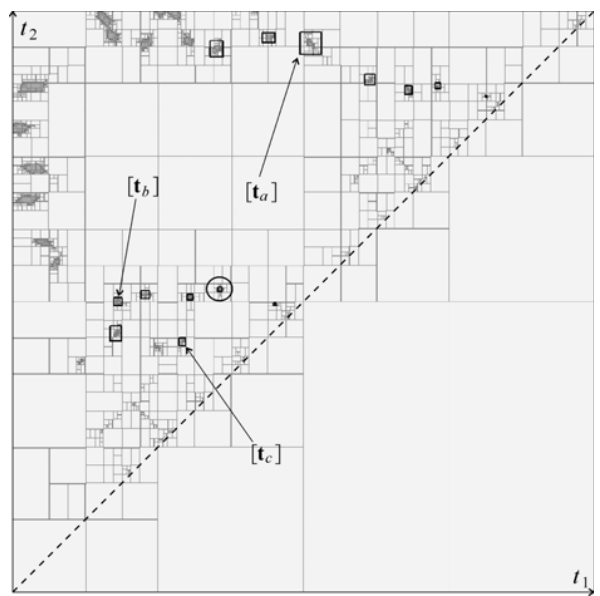

Fig. 7. Inner and outer approximation of $\mathbb{T}$.

$\dot{\mathbf{p}}=\mathbf{v}=\left(\begin{array}{cc}\cos \psi & -\sin \psi \\ \sin \psi & \cos \psi\end{array}\right) \cdot\left(\begin{array}{l}u_{x} \\ u_{y}\end{array}\right)$

where $\left(u_{x}, u_{y}\right)^{T}$ are the coordinates of the robot speed in the robot frame and $\psi$ is the heading angle. The initial position $\mathbf{p}(0)$ corresponds to the origin of the frame. Only $u_{x}, u_{y}, \psi$ are measured and we assume that tubes $\left[u_{x}\right],\left[u_{y}\right],[\psi]$ are available. Using interval arithmetic, we can thus get a tube $[\mathbf{v}]$ for $\mathbf{v}$. Consider for instance an experiment described in Jaulin (2009) where the robot to be considered (see Fig. 5) is a mine hunter, named Redermor. The robot moves on a horizontal plane $20 \mathrm{~m}$ deep and the duration of the mission was about two hours in the Douarnenez bay, in Brittany (France). The actual speed (in meters per second) $\mathbf{u}$ is known to satisfy

$\mathbf{u} \in\left(\begin{array}{l}{\left[0.996 \tilde{u}_{x}-0.004,1.004 \widetilde{u}_{x}+0.004\right]} \\ {\left[0.996 \tilde{u}_{y}-0.004,1.004 \widetilde{u}_{y}+0.004\right]}\end{array}\right)$,

where $\left(\widetilde{u}_{x}, \widetilde{u}_{y}\right)$ denotes the speed returned by the loch-Doppler (Workhorse Navigator Doppler Velocity Log). If $\tilde{\psi}$ is the heading (in radian) returned by our gyrocompass (Octans III from IXSEA), then the actual heading should satisfy $\psi \in \tilde{\psi} \pm 0.00527$. Using an interval integration, we get the tube $\int_{0}^{t}[\mathbf{v}](\tau) d \tau$ of Fig. 6 . Note that since only proprioceptive sensors are assumed to be available, the size of the boxes increases with time. The black curve represents an estimation of the true trajectory obtained by a Kalman smoother with exteroceptive sensors that are not assumed to be available for our loop detection. The two black points correspond to the initial and the final pose of the robot. The algorithm Loop described in Table 1 generates the approximation of Fig. 7 in less than 2 min (for a mission of two hours) with a conventional laptop. The dashed line corresponds to the no-delay line $t_{2}-t_{1}=0$. All loops at the neighborhood of this line correspond to small loops. They are all easily detected with a good accuracy even if $t_{1}, t_{2}$ are large. The Newton test has proved the existence and uniqueness of a loop for 14 disjoint $t$-boxes which are in black in Fig. 7. It means that we have been able to prove the existence of at least 14 loops, whereas there exist 28 loops on the trajectory estimated by the Kalman smoother. Most of these $14 t$-boxes are small and near the no-delay line. Only the largest boxes can be seen, as for instance $\left[\mathbf{t}_{a}\right]$, $\left[\mathbf{t}_{b}\right]$ and $\left[\mathbf{t}_{c}\right]$. Since all boxes on the right and below $\left[\mathbf{t}_{c}\right]$ are inside $\mathbb{T}^{\text {out }}$, we can conclude that the corresponding loop is a small loop. In the same manner, we can also conclude that $\left[\mathbf{t}_{b}\right]$ is a complex loop which encloses the small loop corresponding to [ $\left.\mathbf{t}_{c}\right]$. A spacial approximation of these 14 detected loops is represented 


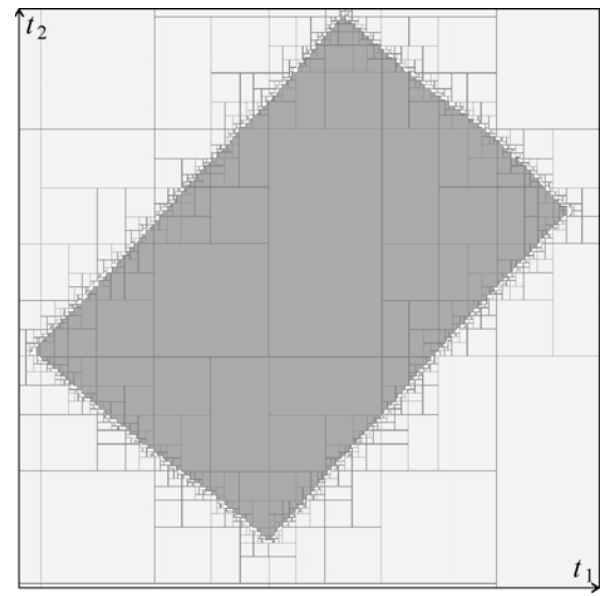

Fig. 8. Zoom of the paving generated by Loop around a feasible zone.

by the thick gray/black crosses of Fig. 6 . The three black crosses correspond to loops $\mathbf{t}_{a}, \mathbf{t}_{b}$, and $\mathbf{t}_{c}$. The black circle of Fig. 7 shows a tiny connected components of $\mathbb{T}$ enclosed by a $t$-box for which the Newton test succeeds. A zoom around this feasible zone is depicted in Fig. 8. The dark gray, light gray and white boxes belong to $\mathbb{T}^{\text {in }}$, $\mathbb{T}^{\text {out }}$ and $\mathbb{T}^{\text {? }}$, respectively. Therefore, we can conclude that the union of all white and dark gray boxes of Fig. 8 contains exactly one loop $\left(t_{1}, t_{2}\right)$. The QT-C++ program with all data and source codes are made available at (Aubry, Desmare, and, \& Jaulin, 2012).

\section{Conclusion}

This paper has presented a new method to detect and localize loops made by a robot during a mission using proprioceptive sensors only. It is only assumed that the robot moves on a 2D environment and that velocity of the robot is known with an error that can be bounded. This is different from the existing loop detection approaches in SLAM (Angel et al., 2008; Joly \& Rives, 2008) which mainly work outs the common features observed from similar points in the scene so that a more accurate estimation of the robot trajectory can be obtained. Since the presented approach does not use scene observations, even if it detects loops it is not able to refine the trajectory envelope. However, our method can help classical SLAM techniques by selecting only $t$-pairs that are consistent with proprioceptive measurements. This decreases the computing burden of the scene-based loop detection and reduces the number of false detections. Moreover, this paper has presented an original formalization of the loop detection problem using the new notion of $t$-space. The introduction of the new concept of integral with interval bounds has made it possible to develop inclusion tests that have been used for the characterization of the set of all feasible loops. The efficiency of the method has been demonstrated on an actual experiment where an underwater robot has performed a mission of two hours with many loops ( 28 for the estimated trajectory).

\section{References}

Abdallah, F., Gning, A., \& Bonnifait, P. (2008). Box particle filtering for nonlinear state estimation using interval analysis. Automatica, 44(3), 807-815.

Angel, A., Filliat, D., Doncieux, S., \& Meyer, J. (2008). A fast and incremental method for loop-closure detection using bags of visual words. IEEE Transactions On Robotics, 24, 1027-1037 (Special Issue on Visual SLAM).

Aubry, C., Desmare, R., \& Jaulin, L. (2012). Programs for loop detection. Available at www.ensta-bretagne.fr/jaulin/loop.html. Ecole navale and ENSTA-Bretagne.
Ben-Zvi, A. (2010). A computationally efficient algorithm for testing the identifiability of polynomial systems with applications to biological systems. Industrial and Engineering Chemistry Research, 49(13), 6125-6134.

Braems, I., Jaulin, L., Kieffer, M., \& Walter, E. (2001). Guaranteed numerical alternatives to structural identifiability testing. In Proceedings of the 40th IEEE conference on decision and control, Vol. 4 (pp. 3122-3127). December. Orlando.

Chabert, G., \& Jaulin, L. (2009). Contractor programming. Artificial Intelligence, 173, 1079-1100.

Davey, B. A., \& Priestley, H. A. (2002). Introduction to lattices and order. Cambridge University Press, ISBN: 0521784514.

Delanoue, N., Jaulin, L., \& Cottenceau, B. (2006). Using interval arithmetic to prove that a set is path-connected. Theoretical Computer Science, 351(1), 119-128 (Special Issue: Real Numbers and Computers).

Drevelle, V., \& Bonnifait, P. (2009). High integrity gnss location zone characterization using interval analysis. In ION GNSS.

Drocourt, C., Delahoche, L., Brassart, B.M.E., \& Clerentin, A. (2005). Incremental construction of the robot's environmental map using interval analysis. Global optimization and constraint satisfaction: second international workshop, Vol. 3478 , COCOS 2003 (pp. 127-141).

Goldsztejn, A., Hayes, W., \& Collins, P. (2011). Tinkerbell is chaotic. SIAM Journal on Applied Dynamical Systems, 10(4), 1480-1501.

Jaulin, L. (2009). A nonlinear set-membership approach for the localization and map building of an underwater robot using interval constraint propagation. IEEE Transaction on Robotics, 25(1), 88-98.

Jaulin, L., Kieffer, M., Didrit, O., \& Walter, E. (2001). Applied interval analysis, with examples in parameter and state estimation, robust control and robotics. London: Springer-Verlag.

Jaulin, L., \& Walter, E. (1993). Guaranteed nonlinear parameter estimation from bounded-error data via interval analysis. Mathematics and Computers in Simulation, 35(2), 123-137.

Joly, C., \& Rives, P. (2008). Bearing-only SLAM: comparison between probabilistic and deterministic methods. Research Report RR-6602, INRIA. URL http://hal.inria.fr/inria-00308722/en/.

Kurzhanski, A., \& Valyi, I. (1997). Ellipsoidal calculus for estimation and control. Boston, MA: Birkhäuser.

Lagrange, S., Delanoue, N., \& Jaulin, L. (2007). On sufficient conditions of injectivity, development of a numerical test via interval analysis. Reliable Computing, 13(5), 409-421.

Lagrange, S., Delanoue, N., \& Jaulin, L. (2008). Injectivity analysis using interval analysis application to structural identifiability. Automatica, 44(11), 2959-2962.

Lapierre, L., Zapata, R., \& Lépinay, P. (2007). Combined path-following and obstacle avoidance control of a wheeled robot. International Journal of Robotics Research, 26(4), 361-375.

LeBars, F., Sliwka, J., Reynet, O., \& Jaulin, L. (2012). State estimation with fleeting data. Automatica, 48(2), 381-387.

Leonard, J. J., \& Durrant-Whyte, H. F. (1992). Dynamic map building for an autonomous mobile robot. International Journal of Robotics Research, 11(4).

Lydoire, F., \& Poignet, P. (2003). Nonlinear predictive control using constraint satisfaction. In 2nd international workshop on global constrained optimization and constraint satisfaction, COCOS (pp. 179-188).

Meizel, D., Preciado-Ruiz, A., \& Halbwachs, E. (1996). Estimation of mobile robot localization: geometric approaches. In M. Milanese, J. Norton, H. Piet-Lahanier, \& E. Walter (Eds.), Bounding approaches to system identification (pp. 463-489). New York, NY: Plenum Press.

Moore, R. E. (1979). Methods and applications of interval analysis. Philadelphia, PA: SIAM.

Raissi, T., Ramdani, N., \& Candau, Y. (2004). Set membership state and parameter estimation for systems described by nonlinear differential equations. Automatica, 40, 1771-1777.

Raksanyi, A., Lecourtier, Y., Walter, E., \& Venot, E. (1985). Identifiability and distinguishability testing in computer algebra. Mathematical Biosciences, $77(1-2), 245-266$.

Sainudiin, R. (2010). Machine interval experiments: accounting for the physical limits on empirical and numerical resolutions. Köln, Germany: LAP Academic Publishers.

Thrun, S., Bugard, W., \& Fox, D. (2005). Probabilistic robotics. Cambridge, MA: MIT Press.

Tucker, W. (1999). The Lorenz attractor exists. Comptes Rendus de l'académie des Sciences, 328(12), 1197-1202.

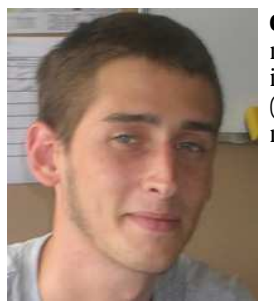

Clément Aubry was born in Laval, France in 1986. He received a M.Sc. in Dynamic Systems and Signals at ISTIA in 2010. He started his Ph.D. studies in 2010 at IRENav (French Naval Academy Research Institute) on interval methods applied to underwater robotics. 


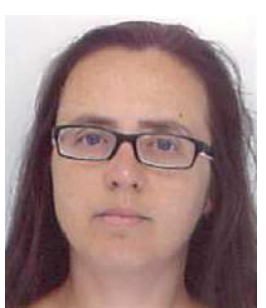

Rozenn Desmare is Associate Professor in Underwater Acoustics Research Team at IRENav (French Naval Academy Research Institute) since September 2000. She received a Ph.D. in Medical Acoustic Imaging from "Université du Maine" in December 1999. Her research concerns piezoelectric transducers, array and sonar system.

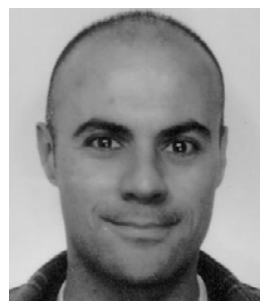

Luc Jaulin was born in Nevers, France in 1967. He received the Ph.D. degree in automatic control from the University of Orsay, France in 1993. He is currently Professor of Robotics at the ENSIETA engineering school Brest, France from 2004 . He does his research on underwater robotics using interval methods and constraint propagation. 